\title{
Primary Pancreatic Mantle Cell Lymphoma Diagnosed via Endoscopic Ultrasound-Guided Fine-Needle Aspiration
}

\author{
Takuo Yamai $^{a}$ Kenji Ikezawa ${ }^{a} K$ Kazuma Daiku ${ }^{a}$ Shingo Maeda ${ }^{a}$ \\ Yutaro Abe ${ }^{a}$ Yugo kai ${ }^{a}$ Ryoji Takada ${ }^{a}$ Tasuku Nakabori ${ }^{a}$ \\ Nobuyasu Fukutake $^{a}$ Hiroyuki Uehara $^{a}$ Hiroaki Masaie $^{b}$ Kaori Ishida $^{c}$ \\ Chiaki Kubo $^{d}$ Kazuyoshi Ohkawa ${ }^{a}$ \\ aDepartment of Hepatobiliary and Pancreatic Oncology, Osaka International Cancer \\ Institute, Osaka, Japan; ${ }^{b}$ Department of Hematology, Osaka International Cancer Institute, \\ Osaka, Japan; 'Department of Clinical Laboratory, Osaka International Cancer Institute, \\ Osaka, Japan; dDepartment of Diagnostic Pathology and Cytology, Osaka International \\ Cancer Institute, Osaka, Japan
}

\section{Keywords}

Primary pancreatic lymphoma · Endoscopic ultrasound-guided fine-needle aspiration .

Pancreatic tumor

\section{Abstract}

Primary pancreatic lymphomas (PPLs) are rare, and the histological classification of these tumors is difficult. To accurately diagnose and determine the appropriate treatment for PPLs, sufficient sample amounts are necessary. Here, we report a 73-year-old man with a primary pancreatic mantle cell lymphoma. Histological samples were obtained via endoscopic ultrasound-guided fine-needle aspiration (EUS-FNA). The tumor cells predominantly composed of atypical small to medium round cells, with diffuse immunoreactivity of CD20 and cyclin D1. In addition, immunoglobulin gene $\mathrm{H}$ chain rearrangement was detected. The patient underwent chemotherapy, resulting in complete remission. Eight years after the initiation of chemotherapy, the patient was still alive. EUS-FNA could be a useful and safe diagnostic modality for PPLs by providing enough samples for testing. 
Fig. 1. Abdominal CECT images. Irregular contour of the pancreas was observed (arrowhead). CECT, contrast-enhanced computed tomography.

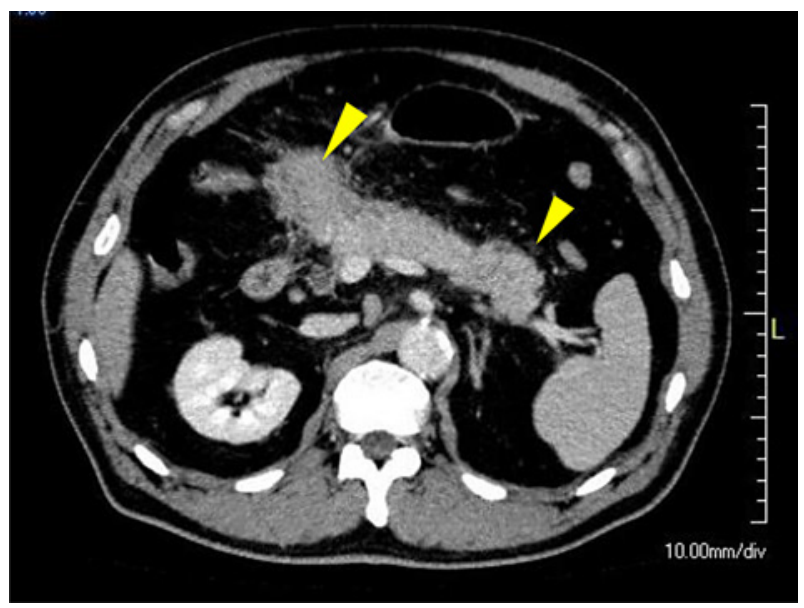

\section{Introduction}

Primary pancreatic lymphomas (PPLs) account for less than $2 \%$ of all extranodal lymphomas and $0.5 \%$ of all pancreatic masses [1]. A lymphomatous mass localized to the pancreas may be diagnosed as a PPL, which typically presents as a large bulky mass in the pancreatic head [2]. A previous report showed that the median diameter of PPLs was $7.9 \mathrm{~cm}$ (range 2.4-13.9 cm) in 44 patients [3]. Chemotherapy for PPLs achieves a high complete response rate [3]; however, accurate pathological diagnosis requires a sufficient sample amount. Tumor biopsy by laparotomy obtains enough samples but may be relatively invasive. Although endoscopic ultrasound-guided fine-needle aspiration (EUS-FNA) is a less-invasive modality for tissue acquisition of pancreatic tumors [4], the effectiveness of EUS-FNA for the diagnosis of PPLs remains unclear. Here, we report a rare case of a pancreatic mantle cell lymphoma in which the use of EUS-FNA contributed to an accurate diagnosis and the selection of an efficient treatment.

\section{Case Presentation}

A 73-year-old man underwent transabdominal ultrasonography during a follow-up examination for fatty liver disease; the procedure unexpectedly revealed a pancreatic mass. He had no history of cancer, smoking, or alcohol consumption. His physical examination revealed that his vital signs were normal, and his abdominal examination was unremarkable. Examinations of peripheral blood and biochemistry including hepatobiliary and pancreatic enzymes and tumor markers (carcinoembryonic antigen, carbohydrate antigen 19-9 and DUPAN-2) were within the normal range.

Abdominal contrast-enhanced computed tomography (CT) revealed masses located in the pancreatic head and tail with faint enhancement, while atrophy of the pancreatic parenchyma or dilation of the main pancreatic duct was not observed (Fig. 1). Fluorine-18-fluorodeoxyglucose (FDG) positron emission tomography (PET)-CT demonstrated FDG uptake in the whole pancreas (maximum standardized uptake value: 5.4) (Fig. 2). EUS revealed that the pancreatic parenchyma showed decreased echogenicity and an indistinctly demarcated, internally heterogeneous pancreatic head tumor with enlarged para-aortic lymph nodes (Fig. 3a). To evaluate the pancreatic head mass, EUS-FNA with a 25-gauge needle was performed without complications. The tumor cells were primarily composed of atypical small to medium round cells with a high nuclear/cytoplasmic ratio, while Hodgkin cells or Reed-Sternberg 
Fig. 2. FDG PET-CT images. FDG PET-CT showed FDG uptake in the whole pancreas (SUV max of 5.4, arrowhead). FDG, fluorine18-fluorodeoxyglucose; PET-CT, positron emission tomography/computed tomography; SUVmax, maximum standard uptake value.

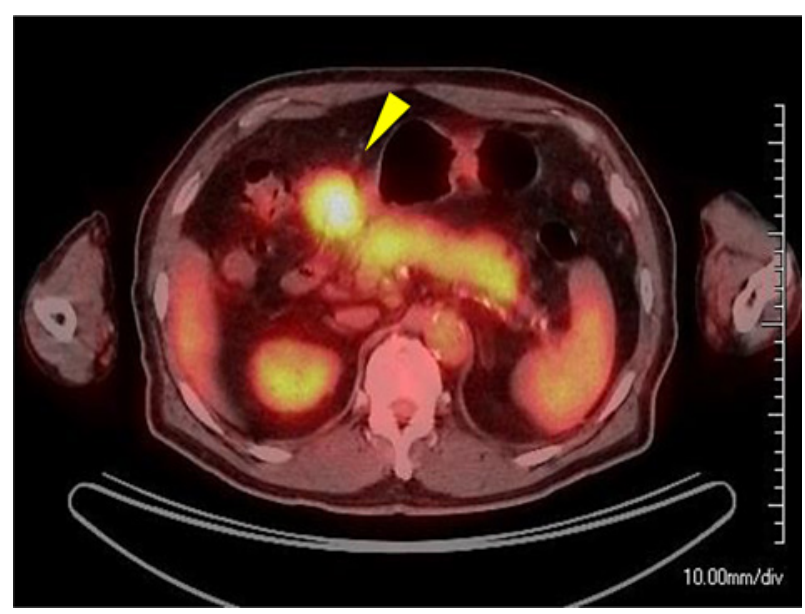

cells were not observed (Fig. 3b, c). CD20 immunoreactivity and cyclin D1 immunoreactivity were diffusely observed (Fig. 3d, e). Additionally, clonality analysis with multiplex PCR assays standardized for the detection of clonally rearranged immunoglobulin and T-cell receptor genes revealed immunoglobulin gene $\mathrm{H}$ chain rearrangement [5]. Collectively, these results led to the final diagnosis of a low-risk mantle cell lymphoma according to the International Prognostic Index. He underwent the rituximab, pirarubicin, cyclophosphamide, vincristine, and prednisolone (R-THP-COP) regimen for 9 months, resulting in complete remission. One year and 9 months after termination of first-line chemotherapy, PET-CT revealed FDG uptake in the pancreas and para-aortic lymph nodes, which was diagnosed as recurrence. With the administration of the rituximab-bendamustine regimen as a second-line chemotherapy, he achieved complete remission again. Eight years after the initiation of first-line chemotherapy, the patient was doing well without recurrence.

\section{Discussion}

We report a case of a patient who achieved long-term survival after chemotherapy based on an accurate pathological diagnosis of a mantle cell lymphoma via EUS-FNA. A PPL is primarily localized in the head of the pancreas as a bulky mass [6] presumably because more lymphoid tissues are distributed in the head of the pancreas [7]. Berhns et al. [8] proposed the following criteria for PPLs:

1. No palpable superficial lymphadenopathy.

2. No enlargement of mediastinal lymph nodes.

3. Normal leukocyte count.

4. At laparotomy, the pancreatic mass predominates with grossly involved lymph nodes confined to the peripancreatic region.

5. No hepatic or splenic involvement.

Taken together with the imaging findings, the patient supposedly satisfied the criteria described above. The contrast-enhanced CT image indicated a slightly contrast-enhanced tumor confined to the head and tail of the pancreas. On the other hand, PET-CT scans revealed FDG accumulation in the whole pancreas, and EUS images showed pancreatic head tumors with extrapancreatic extension and decreased echogenicity in the whole pancreas. These findings suggested that the lymphoma cells had infiltrated the entire pancreas.

Clinical symptoms derived from a PPL were not exhibited in the present case. The clinical subjective symptoms of a PPL are reported as epigastric discomfort, nausea, fatigue and 


\section{Case Reports in Gastroenterology}
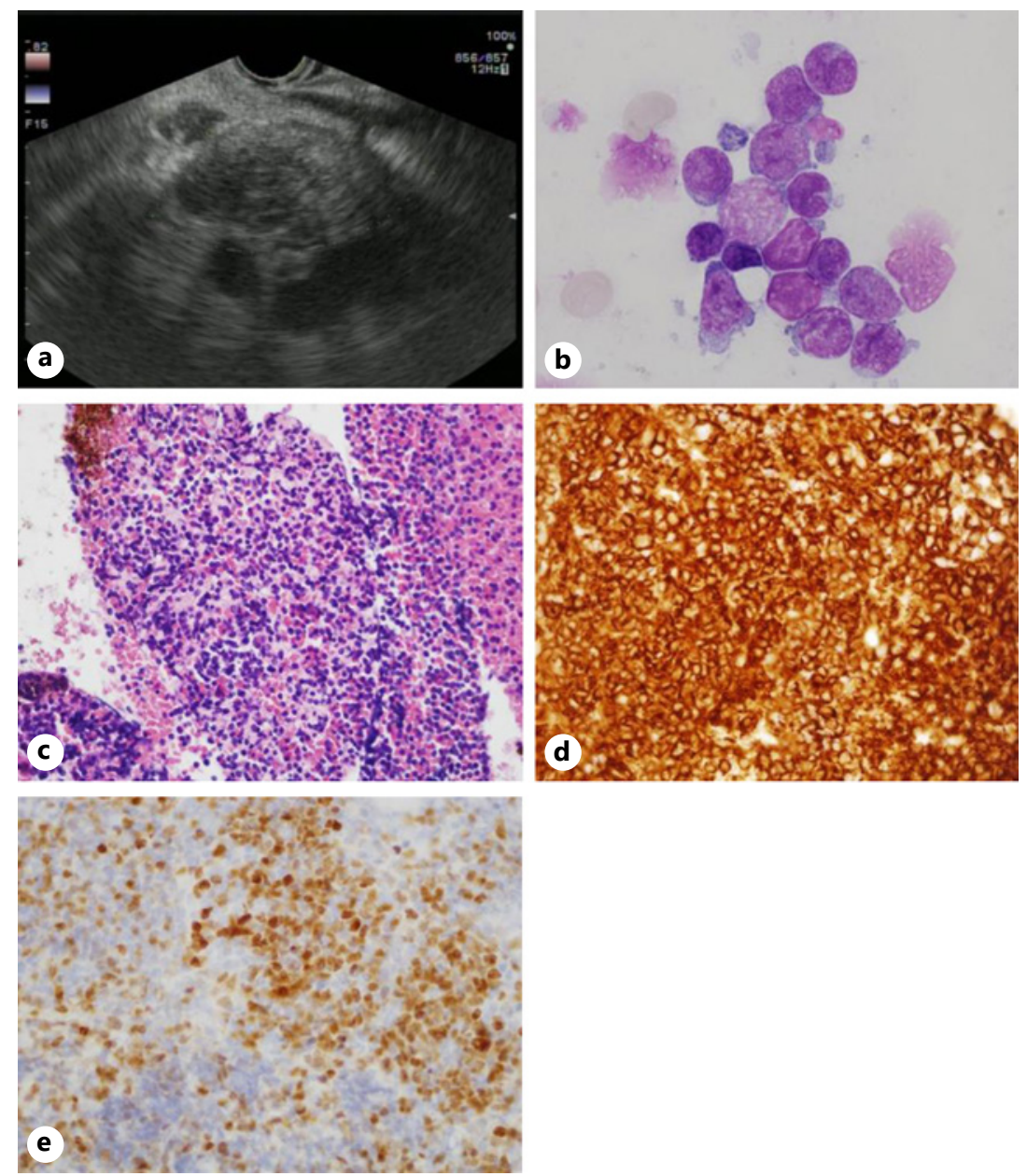

Fig. 3. Pathological examination with samples obtained via EUS-FNA. a EUS image showing the pancreatic head lesion as an indistinct bordered, internally heterogeneous, and hypoechoic mass. b Cytological specimen staining for Giemsa stain (magnification, ×400). c-e Immunohistochemical staining for hematoxylin-eosin (c), CD20 (d), and cyclin D1 (e) (magnification, $\times 400$ ). EUS, endoscopic ultrasound-guided fine-needle aspiration.

anorexia [3]. PPLs are rarely associated with severe fever, night sweats, and weight loss, which are often described in other lymphomas (i.e., B symptoms) [9]. The present case was asymptotic and incidentally detected with abdominal ultrasonography examination at the time of follow-up for the primary disease.

EUS-FNA has been established as an efficient diagnostic technique in the differential diagnosis of pancreatic diseases [10]. Although a meta-analysis demonstrated that EUS-FNA for solid pancreatic tumors had a high sensitivity (86.8\%) and specificity of $95.8 \%$ [11], it has been reported that histological examination with EUS-FNA is not suitable for the diagnosis of PPLs because of the difficulty in obtaining an adequate sample amount [12]. A sufficient amount of sample is required for the definitive diagnosis of PPLs compared to other pancreatic tumors, such as pancreatic ductal adenocarcinoma because compared to cytology alone, flow cytometry and immunohistochemistry enhance the diagnostic efficiency for PPLs [13]. Although laparoscopic biopsies have been performed for the pathological examination of PPLs, laparoscopic examination is an invasive modality because it needs general anesthesia with a long procedure time (approximately 1-4 h) [14]. On the other hand, EUS-FNA is a lessinvasive modality because it does not require general anesthesia and has a shorter procedure 
time [15]. In our case, we successfully performed immunostaining and multiplex PCR assays using specimens obtained via EUS-FNA, suggesting that it could be an efficient and safe modality for the definitive diagnosis of PPLs.

In conclusion, we reported a rare case of a primary pancreatic mantle cell lymphoma diagnosed via EUS-FNA, which provided a sufficient amount of sample not only for histological examinations but also for clonality analysis. Accurate histological findings helped to avoid unnecessary surgery and contribute to the selection of appropriate treatments. EUS-FNA could be a useful and safe diagnostic modality even in cases where the differential diagnosis of a PPL is considered.

\section{Statement of Ethics}

Written informed consent was obtained from the patient for publication of this case report and any accompanying images.

\section{Conflict of Interest Statement}

The authors disclose no conflicts of interest.

\section{Funding Sources}

No specific funding has been received for this study.

\section{Author Contributions}

Study concept and design: T.Y. and K.I. Acquisition of data: T.Y., Y.A., H.M., and C.K. Analysis and interpretation of data: T.Y., K.I., K.D., S.M., Y.A., Y.K., R.T., T.N., N.F., H.U., Y.A., H.M., K.I., C.K., and K.O. Drafting of the manuscript: T.Y. and K.I. Critical revision of the manuscript for important intellectual content: H.M., C.K., and K.O. Final approval of the manuscript: all authors.

\section{References}

1 Teefey SA, Stephens DH, Sheedy PF. CT appearance of primary pancreatic lymphoma. Gastrointest Radiol. 1986;11(1):41-3.

2 Tuchek JM, De Jong SA, Pickleman J. Diagnosis, surgical intervention, and prognosis of primary pancreatic lymphoma. Am Surg. 1993 Aug;59(8):513-8.

3 Sadot E, Yahalom J, Do RK, Teruya-Feldstein J, Allen PJ, Gönen M, et al. Clinical features and outcome of primary pancreatic lymphoma. Ann Surg Oncol. 2015 Apr;22(4):1176-84.

4 Mangiavillano B, Sosa-Valencia L, Deprez P, Eisendrath P, Robles-Medranda C, Eusebi LH, et al. Tissue acquisition and pancreatic masses: Which needle and which acquisition technique should be used? Endosc Int Open. 2020 Oct;8(10):E1315-E20.

5 van Dongen JJ, Langerak AW, Brüggemann M, Evans PA, Hummel M, Lavender FL, et al. Design and standardization of PCR primers and protocols for detection of clonal immunoglobulin and T-cell receptor gene recombinations in suspect lymphoproliferations: report of the BIOMED-2 Concerted Action BMH4-CT98-3936. Leukemia. 2003 Dec;17(12):2257-317.

6 Rad N, Khafaf A, Mohammad Alizadeh AH. Primary pancreatic lymphoma: what we need to know. J Gastrointest Oncol. 2017 Aug;8(4):749-57.

7 Mishra MV, Keith SW, Shen X, Bar AV, Champ CE, Biswas T. Primary pancreatic lymphoma: a population-based analysis using the SEER program. Am J Clin Oncol. 2013 Feb;36(1):38-43.

8 Behrns KE, Sarr MG, Strickler JG. Pancreatic lymphoma: is it a surgical disease? Pancreas. 1994 Sep;9(5):662-7.

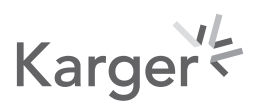


9 Li Z, Zhang S, Vasdani N, Castillo E. Clues for diagnosing primary pancreatic lymphoma. Case Rep Gastroenterol. 2012 May;6(2):438-45.

10 Lai R, Stanley MW, Bardales R, Linzie B, Mallery S. Endoscopic ultrasound-guided pancreatic duct aspiration: diagnostic yield and safety. Endoscopy. 2002 Sep;34(9):715-20.

11 Puli SR, Bechtold ML, Buxbaum JL, Eloubeidi MA. How good is endoscopic ultrasound-guided fine-needle aspiration in diagnosing the correct etiology for a solid pancreatic mass?: a meta-analysis and systematic review. Pancreas. 2013 Jan;42(1):20-6.

12 Thomas T, Kaye PV, Ragunath K, Aithal G. Efficacy, safety, and predictive factors for a positive yield of EUSguided Trucut biopsy: a large tertiary referral center experience. Am J Gastroenterol. 2009 Mar;104(3):58491.

13 Johnson EA, Benson ME, Guda N, Pfau PR, Frick TJ, Gopal DV. Differentiating primary pancreatic lymphoma from adenocarcinoma using endoscopic ultrasound characteristics and flow cytometry: a case-control study. Endosc Ultrasound. 2014 Oct;3(4):221-5.

14 Strasberg SM, Middleton WD, Teefey SA, McNevin MS, Drebin JA. Management of diagnostic dilemmas of the pancreas by ultrasonographically guided laparoscopic biopsy. Surgery. 1999 Oct;126(4):736-3, discussion 41-3.

15 Facciorusso A, Buccino RV, Muscatiello N. How to measure quality in endoscopic ultrasound. Ann Transl Med. 2018 Jul;6(13):266. 